\title{
IL PROBLEMA DELLE COLTIVAZIONI ILLEGALI, UN OSTACOLO ALL'ATTUAZIONE DEL PROCESSO DI PACE IN COLOMBIA ${ }^{1}$
}

\author{
THE PROBLEM OF ILEGAL CULTIVATION: AN OBSTACLE TO THE PEACE PROCESS IN \\ COLOMBIA
}

Jairo E. Cabrera²

\begin{abstract}
Sommario
La ricostruzione dei territori della Colombia nella fase post-accordo, si dovranno avviare a partire da strategie di Sviluppo Alternativo Integrale che permettano di ricostituire i territori interessati dalle coltivazioni illegali. Firmato l'accordo di pace, non costituirà più terreno fertile per la guerra e per la violenza strutturale causate da una cultura dell'illegalità ben radicata nella società. Se le coltivazioni illegali non spariranno dal territorio, l'attuazione degli accordi di pace risulterà un processo praticamente impossibile da realizzare giacché in questa vicenda cambierebbero semplicemente gli attori, passando da un gruppo ribelle a uno di narcotrafficanti e tutta la violenza e il male derivato da questa attività rimarrebbe radicato nel territorio cosicché la Colombia perderebbe un'opportunità storica di andare realmente avanti nonché di pacificare e riattivare il settore rurale.
\end{abstract}

Parole chiavi: Coltivazione Illegali. Processo di Pace in Colombia. Svillupo Alternativo Integrale. Ricostruzione dei territori.

\begin{abstract}
The rebuilding of the territories of Colombia in the post-agreement, will have to start from the Integral Developmental strategies that will help to replenish the territories affected by illegal crops. If the illegal crops do not disappear from the territory, the implementation of peace agreements will be a process virtually impossible to achieve, because in this affair simply change the actors, ranging from a rebel group to one of narcotrafficers and all the violence and evil derived from this activity would remain rooted in the territory, so that Colombia would lose a historic opportunity to go further and to pacify and reactivate the rural sector.
\end{abstract}

Key Words: Illegal Cultivation. Peace Process in Colombia. Alternative Development. Reconstruction of Territories.

1 Artigo submetido em 05/11/2017, pareceres de análise em 04/12/2017, 05/12/2017 e 13/12/2017, aprovação comunicada em 14/12/2017.

2 Professor do Departamento de Derecho Público da Universidad Católica de Colombia, Doutor pela Universita Degli Studi di Palermo, Mestre em Direito Ambiental pela Universita Degli Studi di Palermo e Mestre em Derecho Público pela Universidad Externado de Colombia. E-mail: <jecabrera@ucatolica.edu.co>. 
Indice: 1. Introduzione. 2. Il fallimento delle risposte dello Stato e la stigmatizzazione del movimento contadino. 3. II problema delle coltivazioni illegali. 4. Conclusioni. 5. Bibliografía.

\section{$1 \quad$ INTRODUZIONE}

A causa della propria conformazione la Colombia è sempre stato un paese chiaramente segnato dai conflitti. A partire dagli atti per l'indipendenza passando per l'imposizione di sistemi di governo con strutture centrali alternate a quelle federali nel XIX secolo, dalla contrapposizione dei partiti conservatore e liberale all'inizio alle diverse correnti di pensiero capitalista e comunista, tutto ciò è stato un "brodo di coltura" per la nascita di una guerra fratricida e insensata che ha portato ad oltre sessant'anni di scontro.

Ciò che si intende evidenziare attraverso questa ricerca, con il proposito di contestualizzare la situazione attuale e successivamente includere azioni di ricostruzione nei territori interessati dal conflitto armato colombiano, è l'analisi sulle origini della guerra in Colombia. Sottolineando le radici rurali, l'abbandono sistematico delle campagne, origine delle dolorose diseguaglianze tra aree urbane e rurali, ha portato gruppi ribelli a levarsi in armi contro uno Stato incapace di trovare soluzioni e alternative alle aree più povere e isolate del territorio nazionale.

Dalla sua indipendenza la Colombia ha adottato la forma di una Repubblica, creando una serie di processi di sviluppo e relazionandosi con pochissime città e territori che generavano attività economiche indispensabili per la sopravvivenza del paese: dal centro della propria capitale fino al nord con i porti di Santa Marta e Cartagena, dimenticando evidentemente lo sviluppo degli altri territori; strutturandosi in amministrazioni essenzialmente centralizzate; anticipando già da allora un'assenza dello Stato che sarebbe durato parecchi decenni e che, senza dubbio, ha ridotto la Colombia ad un paese rurale dal quale ha origine questa triste storia di desolazione, abbandono e opposizione.

Sono stati davvero pochi i presidenti che hanno provato ad adottare riforme strutturali che permettessero di dare priorità al comparto agricolo colombiano. Tuttavia, tale trasformazione avrebbe implicato un cambiamento delle modalità e della proprietà delle terre che, senza alcun dubbio, non avrebbe favorito i grandi possidenti, $\mathrm{i}$ quali, per la maggior parte, rivendicavano le proprietà in quanto 
discendenti degli spagnoli e che nel nome del corona hanno occupato migliaia e migliaia di ettari di terreno lungo tutto il paese.

C'è da sottolineare che una politica che pretenda una:

Trasformazione profonda della struttura della proprietà terriera, cioè della modalità di possesso, appropriazione, usufrutto e gestione della terra, prova in generale a redistribuire $\mathrm{i}$ terreni fra i coloni, i contadini e i lavoratori agricoli, sotto la tutela dello Stato. Così come il possesso della terra è fonte di potere politico, l'espropriazione dei proprietari terrieri genera una nuova correlazione di forze e una nuova struttura di potere nelle campagne (MORET, 2003, p. 22)

È evidente che si iniziano a delineare due estremi opposti nel settore agricolo: da un lato i grandi possidenti con potere e influenza politica, dall'altro i contadini senza terra e abbandonati dal governo nazionale.

Cominciamo dunque ad osservare come un tema così sensibile come la proprietà della terra generi situazioni contrapposte in termini di possesso, uso e redistribuzione, poiché come afferma Morett (2003) la terra è la fonte del potere politico, comprese le colonie che rivendicavano il potere sulla terra nonché il potere politico ed economico di una determinata area. Attualmente anche le grandi famiglie di chiaro lignaggio politico e tradizionale sono proprietarie di migliaia di ettari di terreno nelle zone vicine ai propri luoghi d'origine.

Stano così le cose, con la pressione degli uni verso gli altri e con le diseguaglianze e le violazioni dei diritti dei contadini, si comincia a generare una forma di resistenza negli anelli più deboli della catena e si iniziano a creare dei movimenti per richiamare l'attenzione dello Stato affinché intervenga nella faccenda, procedendo con riforme strutturali per favorire condizioni di equità, opportunità ed uguaglianza così come espresso nella carta costituzionale.

Nonostante quanto detto prima, vedremo come nel mondo rurale siano riuscite a penetrare forme di violenza, le cui origini, cause e, talvolta, soluzioni sono da rintracciarsi nelle campagne e tra i contadini.

Al di là della violenza strutturale in Colombia, della presenza di diseguaglianze sociali, degli abusi del bipartitismo, della rinascita degli scontri da parte dei ribelli, del narcotraffico, del paramilitarismo e dell'agire lento e precario per lungo tempo da parte dello Stato nel risolvere i problemi, si è riusciti a portare lo 
Stato colombiano dall'essere un paese fallito a rappresentare un luogo di speranze e sogni con un futuro di pace.

A questo punto la nostra ricerca acquisisce una rilevanza strategica nel progetto di un paese in ricostruzione e che esce da un conflitto quindi è indispensabile conoscerne la storia, imparare dai propri errori. $\dot{E}$ necessario sottolineare che la ricostruzione dei territori si dovranno avviare a partire da strategie di Sviluppo Alternativo Integrale che permettano di ricostituire i territori interessati dalle coltivazioni illegali: già firmato l'accordo, non costituirà più terreno fertile per la guerra e per la violenza strutturale causate da una cultura dell'illegalità ben radicata nella società. Se le coltivazioni illegali non spariranno dal territorio, l'attuazione degli accordi di pace risulterà un processo praticamente impossibile da realizzare giacché in questa vicenda cambierebbero semplicemente gli attori, passando da un gruppo ribelle a uno di narcotrafficanti e tutta la violenza e il male derivato da questa attività rimarrebbe radicato nel territorio cosicché la Colombia perderebbe un'opportunità storica di andare realmente avanti nonché di pacificare e riattivare il settore rurale.

\section{IL FALLIMENTO DELLE RISPOSTE DELLO STATO E LA STIGMATIZZAZIONE DEL MOVIMENTO CONTADINO}

Agli albori del XX secolo il mondo stava cambiando: nuove ideologie e nuovi atteggiamenti iniziarono a trovare spazio nel dibattito sul modello di Stato; la concezione economica e filosofica che fino ad allora predominava ora si trovava alla pari con le istanze portate dalla rivoluzione industriale. Ovviamente la Colombia non faceva eccezione.

La rivoluzione in Unione Sovietica aveva trionfato portando alla nascita di una nuova potenza e la Colombia non era indifferente a queste nuove correnti di pensiero che perseguivano le rivendicazioni sociali degli oppressi. La storia ci rivela che diversi intellettuali colombiani nel 1919 acclamavano questi nuovi ideali affermando che "gli operai colombiani vogliono formare un nuovo partito che abbia in programma le grandi rivendicazioni socialiste. II liberalismo, con un processo di evoluzione, può rappresentare questo partito socialista". (Revista Credencial Historia, 2005, p.1) 
In effetti, il partito liberale in Colombia agli inizi del XX secolo ebbe come ideologia principale l'ideale socialista, accolto da numerosi intellettuali e leader politici del partito: nomi come Enrique Olaya Herrera, Alfonso López, Eduardo Santos - che successivamente saranno presidenti della Repubblica - affermarono "che il sacro proposito del Partito Liberale, nella propria ricerca del potere, era di strutturare le riforme sociali e accettarono in seno alla propria piattaforma non poche tesi del socialismo sovietico". (MACHADO, 1991, p. 342)

In opposizione a simili ideali, il Partito Conservatore non poteva essere d'accordo con le affermazioni sovversive del bolscevismo, combattendole senza sosta in Parlamento, al Governo, sulla stampa e dai palchi dei comizi.

Nel 1928 il liberalismo - ancora in minoranza al Congresso - aveva reso popolare la propria azione e godeva dell'appoggio delle masse. Gli operai, i quali venivano definiti comunisti dal settore più reazionario del conservatorismo, erano ferventi liberali poiché si rispecchiavano negli editoriali della stampa liberale, nei discorsi dei leader del liberalismo, nell'idea della riforma sociale, loro grande speranza. (MACHADO, 1991, p. 343)

II problema delle iniquità, delle falle nel sistema rurale e urbano, del proletariato e della classe dirigente, si trovava in una fase acuta durante cui la formazione contadina e/o sindacale inizia ad organizzare le lotte per i propri diritti e rivendicazioni.

In questa fase della storia, il Partito Liberale incarnava un simile sentimento e rifletteva la percezione delle lotte operaie e delle rivendicazioni sociali che gli strati più poveri richiedevano al Governo, con l'obiettivo di un'immediata sconfitta del Partito Conservatore per giungere nuovamente al potere.

I nuovi attori sociali nel settore agricolo "esigevano migliori condizioni di lavoro e promossero lotte e mobilitazioni contadine che rifiutavano i latifondi non sfruttati e le forme di lavoro e di asservimento pre-capitaliste". (MACHADO, 1991, p. 343)

Tuttavia il problema, come è già stato accennato, non è sorto in quel preciso momento storico ma in realtà derivava dei tempi della colonizzazione spagnola secondo il modello politico, sociale ed economico imposto per favorire gli spagnoli e i loro discendenti rispetto agli indigeni e ai creoli, diseguaglianze che rimarranno e aumenteranno negli anni successivi. 
L'influenza ideologica delle altre lotte popolari e l'impennata del prezzo del caffè aumentarono la richiesta di terra. Vega (2004) ricorda che:

La sentenza della Corte Suprema di Giustizia del 1926, che obbligò tutti i proprietari terrieri in causa legale a presentare i titoli di proprietà, scatenò nuovi e più intensi conflitti, specialmente nelle aziende agricole di caffe del Sumapaz, del Tequendama e della parte orientale del Tolima poiché i coloni e gli affittuari scorsero l'opportunità di "mettere in discussione ciò che fino ad allora era una intoccabile gestione interna delle aziende agricole.

Le leghe agrarie, come rappresentanti del movimento contadino, portarono a conoscenza, non solo a livello nazionale ma anche internazionale, dei grandi problemi nel settore rurale che la Colombia pativa, specialmente la diseguaglianza latente nella distribuzione della terra per cui il governo liberale prese in mano la situazione con l'obiettivo di "superare l'ostilità e la contrapposizione tra conservatori e liberali e di contenere la violenza nelle zone rurali (MACHADO, 1991, p. 343)

\begin{abstract}
La spirale di violenza e la mancanza di terra portarono i contadini ad esigere una soluzione ai loro problemi con le occupazioni di fatto, attraverso il confronto con lo Stato e i proprietari terrieri, con le associazioni corporative che reclamavano il possesso delle terre che lavoravano e con la fine della gestione dei poderi non sfruttati. In sintesi, lottavano per una riforma agraria. (MACHADO, 1991, p. 343)
\end{abstract}

Alla fine, il clamore e le pressioni dei contadini colombiani, assieme alle ideologie del partito di governo e alla volontà del presidente Alfonso López Pumarejo, fecero promulgare la legge 200 del 1936 il cui obiettivo principale era quello di ordinare e stabilire un sistema adatto per la gestione e lo sfruttamento della terra.

Secondo il ricercatore Balcázar (2001) la legge 200 "contribuì a legalizzare terre riguardo a cui non c'era chiarezza sulla proprietà e contemporaneamente facilitò l'acquisizione di appezzamenti da parte degli affittuari e la legalizzazione del possesso dei coloni".

Nonostante quanto detto, la norma non sortì gli effetti menzionati né rivestì fedelmente il ruolo per il quale era stata concepita poiché essa rafforzò la proprietà della terra attraverso l'affermazione della funzione sociale e inoltre non si riuscì ad ottenere la sua redistribuzione, punto fondamentale dei problemi agrari descritti 
precedentemente. In pratica, non si raggiunse uno sfruttamento sociale adeguato della terra in via formale.

La legge 200 non stabilì che i latifondisti avrebbero modernizzato subito le condizioni produttive per conformare la struttura agraria alle necessità dello sviluppo capitalista ma dispose soltanto la fine del dominio per incentivare l'aumento della produzione per un periodo di dieci anni. D'altra parte, non ordinò che in quel periodo avrebbero dovuto estinguersi le forme di produzione arcaiche ma che il proprietario avrebbe dovuto tentare lo sfruttamento economico del podere. La legge riconosceva che fosse impossibile instaurare immediatamente la produzione capitalista nel settore rurale e accettò il fatto che la forza lavoro dovesse continuare ad essere sfruttata in altra maniera, sempre che i poderi fossero utilizzabili per le attività produttive. La legge "provava a dare alla proprietà rurale il sostegno giuridico di cui era priva. In altre parole, cercava di impedire il disconoscimento progressivo della proprietà latifondiaria. (MACHADO, 1991, p. 434)

A dispetto degli eventi descritti, l'avvento della seconda guerra mondiale ebbe un impatto significativo nello sviluppo rurale del paese, relegando la riforma agraria in secondo piano e dando priorità alla sostituzione delle importazioni di alimenti e materie prime.

II governo del presidente Eduardo Santos a cavallo degli anni 1938 e 1942 mise in moto un piano di incremento della produzione per ridurre i costi delle materie prime e dei prodotti alimentari.

La scarsa efficacia di tale politica contribuì a tenere bloccata l'industrializzazione. Durante il secondo mandato di Alfonso López Pumarejo (1942-1945), fu approvata la legge 100 del 1944 per riattivare la produzione alimentare. Furono accolte quelle forme di produzione proprie delle haciendas e venne affermata la convenienza pubblica nello sfruttamento delle terre attraverso contratti di mezzadria. Proprio come la legge 200, cercava lo sfruttamento della terra senza rendere conto di chi o come. (MACHADO, 1991, p. 434)

Gli effetti delle legge 200 del 1936 furono neutralizzati dalla legge 100 del 1944:

Controriforma che ribaltò l'obiettivo di trasformare il latifondo sostenendo i contratti di mezzadria. La legge 100 cercava di incentivare la produzione di alimenti (per la maggior parte proveniente dall'economia contadina) la cui offerta si era ridotta. Ma al contrario, stimolò l'allevamento estensivo a scapito dello sviluppo agricolo con il conseguente abbandono di mezzadri e coloni. (MACHADO, 1991, p. 434) 
Ciò che realmente interessava al legislatore non era realizzare una redistribuzione della terra né di migliorare la condizione di contadini, coloni e mezzadri, anche perché in fin dei conti a questa norma non interessava chi fosse il proprietario della terra o se lo sfruttamento della stessa si realizzasse con sistemi poco ortodossi in contrasto con gli interessi e i diritti minimi dei contadini. Al legislatore importava semplicemente produrre. Non il come né con chi. Semplicemente produrre, disumanizzando nuovamente l'attività agricola, aggiungendo un nuovo elemento al disagio già esistente verso coloro che difendevano i diritti dei contadini e sostenevano la redistribuzione della terra. In pratica, il conflitto sociale si trovava ad un punto di non ritorno e molto presto sarebbe esploso in modo fino ad allora ritenuto inimmaginabile.

Verso la fine degli anni '40:

Il conflitto sociale agrario aveva raggiunto un certo livello di violenza, sostenuto dalle ideologie di partito di coloro che invocavano il controllo della terra e da chi ne appoggiava la distribuzione sociale. Ciò portò ad un'ondata migratoria dalle campagne verso le città e aggravò la questione giuridica della proprietà a causa dell'abbandono della terra. Lo scontro politico armato compromise la produzione agricola, quella del caffè e il comparto industriale, riducendo l'offerta alimentare e creando più disoccupazione. (MACHADO, 1991, p. 434)

II destino del paese era già scritto: il conflitto stava per scoppiare in tutta la sua intensità, il crollo del governo era imminente dinanzi all'impossibilità di dare risposte alle necessità dei contadini. Fu del tutto inutile modificare la struttura del potere in favore del settore agricolo nonché l'ascesa di una nuova prosperosa classe sociale di estrazione rurale attraverso l'inclusione e la riduzione del divario in termini di povertà e di iniquità che la separava dai centri urbani.

Anche se la riforma agraria potè rappresentare uno strumento efficace per dare avvio a politiche di sviluppo della prosperità e del benessere:

La Colombia si è dedicata negli ultimi quaranta anni a realizzare un modello di sviluppo rurale senza accesso alla proprietà stessa e con scarsa capacità nel garantire i diritti di possesso. Si verificò dunque uno sviluppo rurale strumentale con approccio produttivo ma senza una strategia di trasformazione delle strutture politiche e sociali del mondo contadino e ancor meno senza una proposta di articolazione strutturata, equa e sostenibile tra la campagna e la città. L'urbano e il rurale si comportavano come due poli opposti e contraddittori invece di rappresentare una coppia articolata che poteva potenziare lo sviluppo senza conflitto. II criterio di consegna delle terre ad alto costo ai contadini con alto indice di analfabetismo, senza reti 
sociali organizzate, privi di capitale sociale, serviti da organismi pubblici con alto livello di inefficienza e corruzione, attuato con principi centralistici e a difesa di interessi non pubblici, terminò con la possibilità di trasformare il settore agricolo. (Credencial Historia, 1999, p. 34)

Da allora la Colombia abbandonò l'ambito rurale e diede priorità ad altro tipo di politica urbana. Nonostante fosse un paese principalmente agicolo, disconosceva la realtà delle campagne colombiane e lasciò terreno fertile per dare inizio a tutti i tipi di conflitto: le carte erano in tavola ma purtroppo il settore rurale è stato ed ha continuato ad essere l'epicentro della guerra. II conflitto nasce, cresce e sopravvive grazie all'iniquità sociale, al divario abissale, all'oblio e all'abbandono che dalla sua creazione come paese ha portato il Governo centrale in campo.

\section{IL PROBLEMA DELLE COLTIVAZIONI ILLEGALI}

Lo sconforto dei colombiani era crescente, il nuovo millennio non recava con sé alcuna speranza, la guerra, che originariamente si era sviluppata in ambienti rurali, per la prima volta si avvicinava alle città. II narcotraffico aveva portato il terrore agli abitanti delle città, la sofferenza e la capacità della guerriglia di conquistare intere città erano all'ordine del giorno.

II conflitto che diventava sempre più intenso, l'iniziativa condotta dalla guerriglia, i grandi colpi inferti ad un esercito non professionale, senza alcun sostegno organizzativo né logistico che portasse benefici strategici dipingevano uno scenario negativo per il paese nei primi anni del XXI secolo.

In definitiva, il paese si trovava sotto assedio e, per la prima volta, apparve concreta la possibilità che la guerriglia potesse conquistare il potere con le armi visto che si trovava a pochi chilometri da Bogotá, la capitale.

\footnotetext{
Mentre nelle aree rurali si verificavano opportunità e cambiamenti nel mezzo di una disputa territoriale feroce fra guerriglia e gruppi paramilitari, il conflitto armato si è sviluppato all'interno di una profonda crisi economica. II periodo di crescita economica che va dal 1993 al 1995 ha lasciato il passo a un periodo di rallentamento tra il 1996 e il 1997 che si è trasformato in crisi tra il 1998 e il 2002, registrando anche una forte decrescita economica nel 1999 (il PIL diminuì del $4,2 \%$ ), il tutto aggravato da un tasso di disoccupazione che è passato dal $18 \%$ al $19,5 \%$ tra gli anni $1999-2002$ quando invece era ad una sola cifra nel periodo 1991-1995. Questo peggioramento della situazione economica generale della popolazione ha inciso notevolmente nel cambiamento della percezione dell'opinione pubblica riguardo al conflitto armato e al processo di pace. Infatti sparì dalla circolazione la frase fatta che
} 
per tanti anni era stata presente nella società colombiana secondo cui "l'economia poteva andar bene nonostante il paese andasse a male. (Ufficio delle nazioni Unite per il controllo della droga e la prevenzione del crimine UNODC, 2014)

L'opinione pubblica ha iniziato allora a percepire come il conflitto armato aggravasse la crisi economica che ha inciso sulla radicalizzazione politica della gente, facendola orientare su una soluzione militare della guerra.

\begin{abstract}
Il business delle droghe illegali che comprende le diverse fasi di coltivazione, lavorazione, esportazione, distribuzione e riciclaggio di denaro. Questo non mette solamente in pericolo la stabilità del paese e la coesione della società ma provoca danni all'economia, indebolisce le istituzioni, alimenta la corruzione, stravolge i valori e provoca la morte di migliaia di giudici, giornalisti, politici, poliziotti e soldati. È cambiata anche la fonte primaria di risorse per finanziare le attività terroristiche delle organizzazioni armate criminali e la minaccia alla biodiversità della Colombia, una delle più ricche del pianeta (UNODC, 2014, p. 34)
\end{abstract}

La guerra in Colombia risulta difficile da capire visto l'insieme di fattori che sono stati amplificati e che hanno rivolto le attenzioni sulla complicata rete di violenza. Fattori che si articolano ed evidenziano sempre di più nella povertà e nella disuguaglianza, nel conflitto agrario, nella mancanza di unità locale e di alternative, nell'abbandono da parte dello Stato, nell'istituzionalizzazione delle terre, nell'accumulo indebito di terreni, nel business del narcotraffico, nei soldi facili, nelle guerre per il controllo del territorio per gestire gli affari illeciti delle miniere e del narcotraffico, nel controllo del potere, nella corruzione e infine tutti gli altri problemi che non solo altro che benzina sul fuoco della violenza in Colombia. Però una prospettiva di pace con uno dei gruppi più forti potrebbe rappresentare, per la prima volta in molti anni, la svolta per cambiare la rotta di un paese che sia meno violento, più equo e ricco.

Tuttavia, per anni lo Stato non è stato efficiente nel proporre dei piani programmatici e dei progetti indirizzati ad una completa attenzione nei confronti del settore agricolo, ragion per cui una delle alternative economiche che ha dato risultati, specie per tutte le cause descritte nella presente ricerca, ha a che fare con il massimo dell'espansione e della sostenibilità del narcotraffico in tutte le sue dimensioni. In particolare, verso quella che riguarda la popolazione rurale coinvolta nel processo di semina e raccolta.

In accordo col censimento annuale delle coltivazioni di coca in Colombia condotto dall'Ufficio delle Nazioni Unite per il Controllo della Droga e la Prevenzione 
del Crimine (UNODC), nonostante la lotta dello Stato colombiano contro l'espansione delle coltivazioni illegali nel 2016 persistono ancora più146 mila ettari di campi di coca in Colombia, distribuiti in 24 dei 32 dipartimenti del paese ${ }^{108}$, tuttavia appare ancora come una grande sfida per la società colombiana visto che il business è ancora presente e tendente a crescere.

Alcuni autori - come visto all'inizio di questa ricerca - concordano nell'affermare che la violenza e la difficile situazione sociale ed economica della campagna colombiana hanno generato un ambiente favorevole alla comparsa e alla sostenibilità delle coltivazioni illegali in Colombia.

Allo stesso modo, ha creato un clima adatto ai cambiamenti e alle trasformazioni nella struttura agraria e nelle dinamiche economiche e sociali dei suoi abitanti. A loro volta, le coltivazioni illegali potenziano e accelerano queste trasformazioni producendo così un complesso sistema di causa-effetto (Ortiz, 2003) alimentando un circolo vizioso che spinge milioni di colombiani verso un nuovo baratro fatto di povertà e di violazione dei propri diritti.

Con il tempo, queste coltivazioni illegali sono diventate gradualmente l'attività economica principale per migliaia di famiglie colombiane, incrementando i livelli del conflitto, indebolendo il tessuto sociale e cancellando l'identità delle comunità contadine.

Come parte di queste strategie, per evitare il controllo delle autorità, i coltivatori hanno progressivamente diminuito la grandezza dei campi di coca, provocando una maggiore dispersione delle colture. Nonostante questa riduzione e l'espansione di nuove coltivazioni in prossimità delle aree di influenza della coca, le zone di concentrazione hanno resistito nel tempo, il che lascia supporre una relativa stabilità nelle aree di coltura.

\footnotetext{
Nonostante gli elevati guadagni che si generano grazie al business delle droghe illegali, il reddito medio pro capite dei coltivatori non supera i 3.000 dollari trovandosi sotto la cifra considerata come indicatore della soglia di povertà (3.856 dollari) che significa un alto livello di vulnerabilità socioeconomica per queste popolazioni. Considerato che i gruppi armati fuorilegge detengono il controllo delle aree dove sono ubicate le coltivazioni illegali, ciò si traduce in un fattore di rischio per la sopravvivenza di numerose comunità contadine indigene e afrodiscendenti in tutto il territorio nazionale (CÁRDENAS, 2001)
}

In accordo con l'UNODC (2014): 
In Colombia esistono 63.660 famiglie legate alle coltivazioni illegali. Queste comunità hanno difficoltà ad ottenere guadagni leciti perché non cooperano, non dispongono di conoscenze tecniche per migliorare la produzione, non posseggono capitali per avviare imprese, nelle loro proprietà non c'è lo spazio minimo per realizzare i propri progetti e nelle zone di produzione mancano le infrastrutture per commercializzare i prodotti. A ciò va aggiunto che l'offerta dello Stato per lo sviluppo rurale, attraverso meccanismi di crescita e prestiti, esige garanzie, capacità di formulazione di progetti e la presenza di quest'ultimi nella fase di produzione: requisiti che in generale le famiglie legate alle coltivazioni illegali non sono in grado di soddisfare.

Sebbene siano stati attuati programmi di sviluppo alternativo a livello nazionale, "è stato sottolineata la necessità di articolarli con le strategie di controllo istituzionale del territorio, vista l'importanza che riveste la sicurezza per la sostenibilità dei processi promossi insieme alle comunità. $\dot{E}$ importante che i procedimenti di sradicamento manuale siano accompagnati da un'offerta sociale da parte dello Stato per assicurare la completezza dell'intervento.

In questo senso, l'efficacia delle strategie di sradicamento e i programmi di sviluppo alternativo per controllare l'espansione delle coltivazioni illegali non sono stati sufficienti. Infatti i risultati parlano da soli: l'aumento delle coltivazioni non deve essere associata all'assenza della politica ma a dei presupposti limitanti e interventi che tali strategie già stabiliscono. Le aree di maggior presenza delle coltivazioni rimangono isolate dal resto del paese, senza offerta di beni e servizi e con la gente sottoposta a privazioni che impediscono di condurre una vita in condizioni giuste e dignitose. Perciò il business del narcotraffico, malgrado il punto 4 della trattativa con le FARC, continuerà e si intensificherà anche dopo la firma degli accordi. Infatti, nuovi soggetti andranno a sostituire il vuoto lasciato dai gruppi ribelli con lo Stato che dovrà prenderli in considerazione. Però se la ricostruzione dei territori non viene attuata in maniera totale e con un approccio differente riguardo al problema della coltivazioni illegali, la realizzazione del processo di pace sarà praticamente impossibile.

Lo Stato Colombiano per oltre 50 anni ha cercato di realizzare degli interventi sul territorio con l'obiettivo di cambiare le dinamiche del settore rurale. Tuttavia tali operazioni sono state solo abbozzate e ritenute insufficienti poiché non ha avuto la forza e la completezza che consenta di trasformare le aree rurali, considerando che 
ancora oggi sono presenti gli stessi problemi, gli stessi fattori e le stesse criticità che hanno dato luogo e mantenuto il conflitto.

La Politica di Consolidamento e Ricostruzione Territoriale, che sosteneva la politica contro le coltivazioni illegali, non possedeva un'articolazione adeguata sul territorio poiché la maggior parte delle aree che erano oggetto della Politica Pubblica non hanno visto diminuire le proprie colture. Così è stato dimostrato che gli interventi di Sviluppo Alternativo sono stati importanti ma insufficienti di fronte alle situazioni legate il business del narcotraffico.

La seconda fase d'intervento della Politica di Consolidamento prevede l'attività di ricostruzione dei territori approfittando del contesto di pace. Tuttavia la politica commette un errore nel ricostruire le aree incrementando le coltivazioni illegali poiché agire senza colpire tale piaga farà sì che lo Stato ripeta quanto fatto in passato, senza ottenere risultati soddisfacenti che consentano di trasformare le zone rurali: le coltivazioni illegali diventano un ostacolo insormontabile per l'attuazione degli accordi di pace.

L'avvio del processo di pace in Colombia sarà rappresentato dalla visibilità data alle violenze nascoste dal conflitto armato. Per questo motivo, nonostante il business del narcotraffico sia evidente soprattutto riguardo alle coltivazioni illegali che hanno apportato una cultura dell'illegalità e l'odio in alcune zone, qualsiasi intervento integrale che non tenda a diminuire le colture, ad eseguire i programmi di Sviluppo Alternativo e a realizzare una ricostruzione del tessuto umano non otterrà i risultati sperati di mutamento del territorio che possano attenuare questo dramma e contribuire alla costruzione della pace.

La ricostruzione del territorio deve tener conto inizialmente - prima dell'intervento completo dello Stato - di processi di attenzione differenziata nelle aree con presenza di coltivazioni illegali attraverso lo Sviluppo Alternativo Integrale, sempre accompagnato da procedimenti di ricostruzione comunitari e da parte del tessuto sociale.

Nelle comunità colpite dalle coltivazioni illegali, in uno scenario post-conflitto, è indispensabile rafforzare l'autostima, recuperare il senso di appartenenza ad una comunità, creare o riscoprire spazi di interesse collettivo per la vita quotidiana, plasmare e consolidare capacità per la gestione degli interessi collettivi. Così, i servizi sociali devono fungere da catalizzatori di relazioni umane dirette, scoprendo luoghi di interesse collettivo, offrendo occasioni per stabilire relazioni fra persone che 
risiedono nei dintorni; generando sentimenti di comunità e di solidarietà e riscoprendo il piacere della convivenza. Infatti è evidente che non è possibile predeterminare tutto ciò che la ricostruzione di un territorio implica, non solo riguardo agli aspetti materiali o tangibili come l'acqua, la luce e le fognature ma anche verso procedimenti intangibili come il sostegno psicosociale alle popolazioni rurali e il ripristino del tessuto sociale.

È fondamentale operare e far comprendere alle comunità, nelle quali verrà attuato lo Sviluppo Alternativo Integrale, come un intervento dello Stato sul campo agevoli il passaggio verso comunità legittime con un ruolo determinante che non si limiti al sostegno di progetti produttivi integrali che comprendono tutti gli anelli della catena produttiva, dalla produzione alla vendita, se non esiste una ricostruzione del tessuto sociale che implichi di parlare dei legami che mantengono coese le comunità in un determinato territorio. II tessuto sociale lacerato deve mirare all'insieme delle relazioni sociali armoniose degli individui che in quanto tali costituiscono reti e creano relazioni di predominio, di potere, di interesse e di affetto. Tutto ciò per assicurare la produzione e la riproduzione delle condizioni biologiche, sociali, culturali, ambientali ed economiche indispensabili per la nostra permanenza in una zona determinata e soprattutto quando nello stesso territorio si possono incontrare tutti i soggetti coinvolti in anni di conflitto, vittime e carnefici insieme nello stesso luogo.

Il processo di pace non cambierà molto le dinamiche territoriali, specialmente in quei luoghi afflitti dalle coltivazioni illegali. Infatti, le cause del narcotraffico non possono essere addebitate esclusivamente alla mancanza di alternative o all'assenza dello Stato ma anche ad una cultura dell'illegalità e del denaro facile vigente sin dagli anni '80, in pratica due generazioni di colombiani radicate nel territorio. Se non viene ricostruito il tessuto sociale e non viene seguito da uno Sviluppo Alternativo Integrale, i terreni delle colture diventeranno l'ostacolo più grande alla realizzazione dell'accordo di pace. Dunque possiamo affermare che la diminuzione delle coltivazioni illegali rappresenta il fattore determinante per il successo o il fallimento del post-conflitto.

BALCÁZAR, Álvaro,. et al. "Colombia: alcances y lecciones de su experiencia en reforma agraria", Santiago del Cile, CEPAL e Nazioni Unite, 2001. 
BERRY, A. “¿Colombia encontró por fin una reforma agraria que funcione?”, Rivista di Economía Istituzionale 4, 6, 2002.

CÁRDENAS, M., (2001). Economic Growth in Colombia: A reversal of 'Fortune'? .CID Working Paper $\mathrm{N}^{\circ} 83$.

Centro de Memoria Histórica. "Informe Basta Ya, Los orígenes, las dinámicas y el crecimiento del conflicto armado", Bogotá, 2014.

CHISTOPHER Abel, Política, iglesia y partidos en Colombia, Bogotá: FAES/Università Nazionale della Colombia, 1987.

Daniel PÉCAUT, "Violencia y política en Colombia. Elementos de reflexión", Medellín: Hombre Nuevo/Università del Valle, 2003.

Fernán GONZÁLEZ, Para leer la política. Ensayos de historia política de Colombia (Bogotá: CINEP, 1997) in Centro Nacional de Memoria Histórica. "Los orígenes, las dinámicas y el crecimiento del conflicto armado" Bogotá, 2014. Jesús Antonio BEJARANO, "La política de paz durante la administración Barco", en El Gobierno Barco: política, economía y desarrollo social en Colombia, 1986-1990. Bogotá: Fedesarrollo/ Fondo Cultural Cafetero, 1994.

Machado, C. ABSALÓN, Problemas agrarios colombianos", Bogotá, XXI secolo, 1991.

Manuel José Bonnett LOCARNO, "La lucha contra el terrorismo: nueva bipolaridad", in: Consuelo Ahumada y Telma Angarita (eds.), Las políticas de seguridad y sus implicaciones para la región andina, Bogotá, Pontificia Università Javeriana, Facoltà di Scienze Politiche e Relazioni Internazionali, 2005.

Medófilo MEDINA, "La resistencia campesina en el sur de Tolima", in Pasado y presente de la violencia en Colombia, comps. Gonzalo Sánchez e Ricardo Peñaranda Bogotá: Cerec, 1986.

Mindefensa (2007) Política de Consolidación de la Seguridad Democrática. Ministeroo della Difesa Nazionale. Repubblica di Colombia. Tipografia Nazionale.

Morett, S. Jesús, Reforma agraria: del latifundio al neoliberalismo, Messico, Plaza e Valdés, 2003.

Narayan, Deepa. Bonds and Bridges, Social Capital and Poverty. The World Bank. Poverty Reduction and Economic Management Network Poverty Division. Working paper 2167. Washington, 1999. 
Ufficio delle Nazioni Unite contro la droga e il crimine (UNODC). Monitoraggio delle Cotivazioni di Coca 2010.UNODC. Bogotá. Giugno 2011.

Ufficio delle Nazioni Unite contro la droga e il crimine - UNODC, SIMCI. Colombia, Monitoraggio delle Cotivazioni di Coca 2014. Luglio 2015.

Ufficio delle Nazioni Unite contro la droga e il crimine - UNODC, SIMCI. Colombia, Monitoraggio delle Cotivazioni di Coca 2015. Luglio 2016.

Ufficio delle Nazioni Unite contro la droga e il crimine - UNODC, SIMCI. Colombia, Monitoraggio delle Cotivazioni di Coca 2016. Luglio 2017.

Ufficio dell'Alto Consiglio per la Sicurezza Nazionale e Azione Sociale (2011) Propuesta Preliminar para Indicadores de Consolidación PNCRT. Revisione Strategica Politica Nazionale di Consolidamento Territoriale. Giugno 2011.

Ortiz, Cesar Enrique. Cultivos ilícitos y nueva ruralidad en Colombia. Cuadernos de Desarrollo Rural, Num. 50, primo semestre 2003, Pontificia Università Javeriana. Bogotá, Colombia.

Programma delle Nazioni Unite per lo Sviluppo, PNUD "Colombia rural: razones para la esperanza”, 2012, Bogotá.

Rodriguez, Joimer, "el problema agrario en Colombia 1020-2010 - Perspectivas y Dificultades”, Università di Caldas. Sociologia. J. Rodríguez. 2012.

UNODC “Independent Project evaluation of the Alternative Development Programmer in Colombia” Nazioni Unite, New York, November 2014.

http://www.justiciaporcolombia.org/sites/justiciaporcolombia.org/files/u2/onflicto_y_De recho_Internacional_Humanitario_0.pdf

http://www.banrepcultural.org/blaavirtual/biografias/gaitjorg.htm http://www.banrepcultural.org/node/86455 http://www.verdadabierta.com/archivos-para-descargar/category/38-historia1?download=5\%3Ainformede-la-procuradura-general-sobre-el-mas-1983 http://equipopazgobierno.presidencia.gov.co/especiales/abc-del-proceso-depaz/index.html https://www.mesadeconversaciones.com.co/comunicados/comunicado-conjunto-64la-habana-15-de-diciembre-de-2015 http://www.eltiempo.com/politica/proceso-de-paz/proceso-de-paz-encuesta-gallupmuestra-optimismo/16427011 